\title{
Educational standards for training paramedics in ultrasound: a scoping review
}

\author{
Ben Meadley*, Alexander Olaussen, Ashleigh Delorenzo, Nick Roder, Caroline Martin, Toby St. Clair, Andrew Burns,
} Emma Stam and Brett Williams

\begin{abstract}
Background: Paramedic-performed out-of-hospital ultrasound is a novel skill that has gained popularity in some services in recent years. In this setting point-of care ultrasound (POCUS) can provide additional information that can assist with management and guide transport to the most appropriate facility. We sought to explore the different educational approaches used for training paramedics in ultrasound in the out-of-hospital setting.

Methods: Ovid MEDLINE, EMBASE, EBM Reviews, The Cochrane Library, CINAHL plus, The Monash University Research Repository and the British Thesis Library were searched from the $1^{\text {st }}$ of January 1990 to the $6^{\text {th }}$ of April 2016. Google Scholar was searched and reference lists of relevant papers were examined to identify additional studies. Articles were included if they reported on out-of-hospital and POCUS educational approaches for paramedics.

Results: A total of 2002 unique articles were identified of which 18 articles met the inclusion criteria. Most articles reported combined cohorts of emergency providers with varying years of experience though most operators were POCUS naïve. The most common clinical assessment for which paramedic POCUS curricula was described was the focused assessment sonography for trauma (FAST) examination. Education programs varied from two-minutes to two-days with all studies including both didactic and practical training.
\end{abstract}

Conclusion: Education programs for POCUS for paramedics vary considerably, and do not appear to align with qualification level or clinical experience. Further research investigating education and subsequent clinical application of POCUS by paramedics is required, as well as prospective, outcome based studies in order to measure the clinical utility of out-of-hospital POCUS.

Keywords: Out-of-hospital, Paramedic, Ultrasound, Education, Training

\section{Background}

The modern-day Emergency Medical Services (EMS) system is a complex network of coordinated services. Due to the challenging environment and ever-changing nature of work encountered by paramedics, the profession is continually expanding to include a greater range of clinical skills [1]. For example, recent advances have included the addition of point-of-care ultrasound (POCUS) to assist with improved patient outcomes, clinical decision making and triage [2-4]. Balancing over-triage and under-triage is important for the health-care system as a whole and the patient as an individual [5]. Clinical diagnoses alone are

\footnotetext{
*Correspondence: benjamin.meadley@monash.edu Department of Community Emergency Health and Paramedic Practice, Monash University - Peninsula Campus, PO Box 527McMahons Road,

Frankston, VIC 3199, Australia
}

often insufficiently sensitive, leading to misdiagnoses and under-triage of some clinical conditions. In the out-ofhospital setting POCUS can provide additional information, such as earlier detection of intra-abdominal free fluid in patients with blunt trauma, and pericardial effusion in patients with penetrating thoracic trauma $[2,3]$. This information can assist with management strategies, guide transport to the most appropriate facility, and potentially expedite time to definitive intervention [3].

Clinical applications of POCUS in the out-of-hospital setting include enhancing assessment of a wide range of patient cohorts, including but not limited to medical and traumatic patients [2]. For example, the Focused Assessment Sonography for Trauma (FAST) exam is used to detect free fluid in the intra-abdominal compartment [4]. The application of FAST by paramedics, emergency 
physicians and flight nurses has been reported with greater sensitivity and specificity, compared to physical assessment alone, when assessing for hemoperitoneum in patients with penetrating and blunt trauma $[6,7]$.

Moreover, the extended FAST examination (eFAST) also includes thoracic assessment to detect for haemothorax and pneumothorax [8]. This is a time effective procedure particularly when faced with the challenges of auscultating breath sounds in the out-of-hospital environment, such as in a moving ambulance or during aeromedical transport. A diagnostic accuracy of over 90\% when using POCUS to assess for pneumothorax has been reported, suggesting POCUS may be superior to other clinical assessment tools [8]. Correct identification of the presence or absence of pneumothoraces can help guide urgent interventions during resuscitation. Alternatively, this may also prevent unnecessary procedures such as pleural decompression thereby avoiding an invasive procedure that carries potential complications $[9,10]$. Other applications of POCUS performed by paramedics include modified cardiac echocardiography to assess for pericardial fluid and tamponade physiology in the setting of trauma and assessing intra-arrest cardiac wall motion [2,3].

The ability to accurately perform and interpret sonography is likely dependent on appropriate training and education of paramedics [2]. General paramedic training can vary considerably, from vocational basedtraining through to formal tertiary education at the postgraduate level. With regard to demonstrating competence in POCUS, dependence on background education appears most apparent in EMS systems that use non-physician providers, including paramedics and nurses [2]. Most studies report on EMS providers from a range of clinical backgrounds, and evidence pertaining to the clinical utility of paramedic use of POCUS is limited. A systematic review identified multiple training programs with various delivery methods, duration, and assessment [9]. Similarly, El Sayed et al. [2] and Nelson et al. [4] also discussed POCUS curricula for EMS providers in the out-of-hospital setting. These reviews report POCUS to be feasible and time effective with successful application. However, variations in training duration, and the quality of POCUS examinations by different providers are yet to be compared [4]. Presently, it appears there is no agreed curricula standards for the training of paramedics in the provision of out-of-hospital POCUS, making it difficult to draw conclusions regarding the optimal training approach for paramedics.

With the increasing use of out-of-hospital POCUS to assess a number of clinical conditions, it is important to gauge the different methods by which POCUS is currently being taught to paramedics. This will guide which methods suit various EMS agencies with regards to affordability, applicability, and effectiveness. This scoping review aims to explore the different educational approaches used for training paramedics in POCUS in the out-of-hospital setting.

\section{Methods}

We desired to map the available literature relating to paramedics and POCUS education. We therefore chose a scoping review methodology in order to provide a broader understanding of this topic, which can then be narrowed down to a specific research question and systematic review $[11,12]$. In accordance with scoping review practice we included both peer- and non-peer reviewed research, as well as grey literature [12]. We followed the six stage methodology developed by Levac et al. (2010) [12].

\section{Identify the Research Question}

The research question guiding this scoping review was: What educational approaches are used for training paramedics in POCUS? This question was decided upon after initial review of the available literature. The question was felt to strike a balance between being broad enough for a wide selection of papers and at the same time focused enough to generate a search strategy [12].

2. Identify relevant studies

The databases Ovid MEDLINE, EMBASE, EBM Reviews, The Cochrane Library, CINAHL plus, The Monash University Research Repository and the British Thesis Library were searched from the $1^{\text {st }}$ of January 1990 to the $6^{\text {th }}$ of April 2016. The Journal of Medical Sonography and the Air Medical Journal were also searched specifically. In addition, a rigorous search of the grey literature was conducted (Google Scholar, Grey Literature Report and GreyNet International) to identify further studies that might have been missed by the electronic search. A combination of Medical Subject Headings $(\mathrm{MeSH})$ terms and keywords relevant to paramedics and out-of-hospital, POCUS and education (Table 1) were combined using Boolean terms as appropriate. Education interventions were defined as anything featuring teaching, training or practical supervision. We define training in this instance any intervention aimed at increasing competency with use of POCUS.

3. Study selection

Inclusion and exclusion criteria

Studies were eligible for inclusion if they (i) reported on POCUS educational approaches for paramedics and out-of-hospital, and (ii) were published between January 1990 and the $6^{\text {th }}$ of April 2016. This time period was selected because (i) a preliminary review and intimate knowledge of 
Table 1 Summary of search terms combining the 3 search concepts

\begin{tabular}{|c|c|c|}
\hline Paramedics \& Out-of-hospital & Ultrasound & Education \\
\hline emergency medical services/ & ultrasound.mp. & Education/ \\
\hline emergency medical technicians/ & Ultrasonography/ & Teaching/ \\
\hline emergency treatment/ & sonography.mp. & education.mp. \\
\hline emergency medicine/ & Ultrasonography, Doppler, Color/ & Simulation Training/ \\
\hline ambulances/ & Diagnostic Imaging/ & training.mp. \\
\hline air ambulances/ & ultraso\$.mp. & educat\$.mp. \\
\hline retrieval & sonograph\$.mp. & teach\$.mp. \\
\hline HEMS & & train\$.mp. \\
\hline \multicolumn{3}{|l|}{ first aid/ } \\
\hline \multicolumn{3}{|l|}{ military medicine/ } \\
\hline \multicolumn{3}{|l|}{ prehospital } \\
\hline \multicolumn{3}{|l|}{ pre-hospital } \\
\hline \multicolumn{3}{|l|}{ paramedic } \\
\hline \multicolumn{3}{|l|}{ ambulance } \\
\hline \multicolumn{3}{|l|}{ out-of-hospital } \\
\hline \multicolumn{3}{|l|}{ out of hospital } \\
\hline \multicolumn{3}{|l|}{ ems } \\
\hline \multicolumn{3}{|l|}{ emt } \\
\hline \multicolumn{3}{|l|}{ emergency services } \\
\hline \multicolumn{3}{|l|}{ emergency medical service } \\
\hline \multicolumn{3}{|l|}{ emergency technician } \\
\hline \multicolumn{3}{|l|}{ emergency practitioner } \\
\hline \multicolumn{3}{|l|}{ emergency dispatch } \\
\hline \multicolumn{3}{|l|}{ emergency despatch } \\
\hline \multicolumn{3}{|l|}{ first responder } \\
\hline \multicolumn{3}{|l|}{ public access defibrillation } \\
\hline \multicolumn{3}{|l|}{ emergency rescue } \\
\hline \multicolumn{3}{|l|}{ emergency resus } \\
\hline \multicolumn{3}{|l|}{ emergency triage } \\
\hline \multicolumn{3}{|l|}{ advanced life support } \\
\hline \multicolumn{3}{|l|}{ community support co-ordinator } \\
\hline \multicolumn{3}{|l|}{ community support coordinator } \\
\hline \multicolumn{3}{|l|}{ emergency care practitioner } \\
\hline \multicolumn{3}{|l|}{ extended care practitioner } \\
\hline physician assistant & & \\
\hline
\end{tabular}

the field suggested there would not be any relevant articles prior to the year 1990, (ii) cost, and (iii) to only include current and modern methods of education. Studies were excluded if they were not written in English, or if they did not include paramedic providers.

The data bases were searched by one author (AO). Following the search, duplicates were removed and the titles and abstracts subsequently appraised for eligibility by five independent authors (BM,
NR, TSc, CM and BW). The full texts of titles were sourced and reviewed for those studies which were considered potentially relevant. The selection process is described below, see Fig. 1.

4. Charting the data

Charting the data is a 'narrative way' or 'descriptive analytical' method that is used to extract the data from each study [13]. Table 2 provides an overview of the 18 articles selected for inclusion. The key information from the chosen articles were charted 


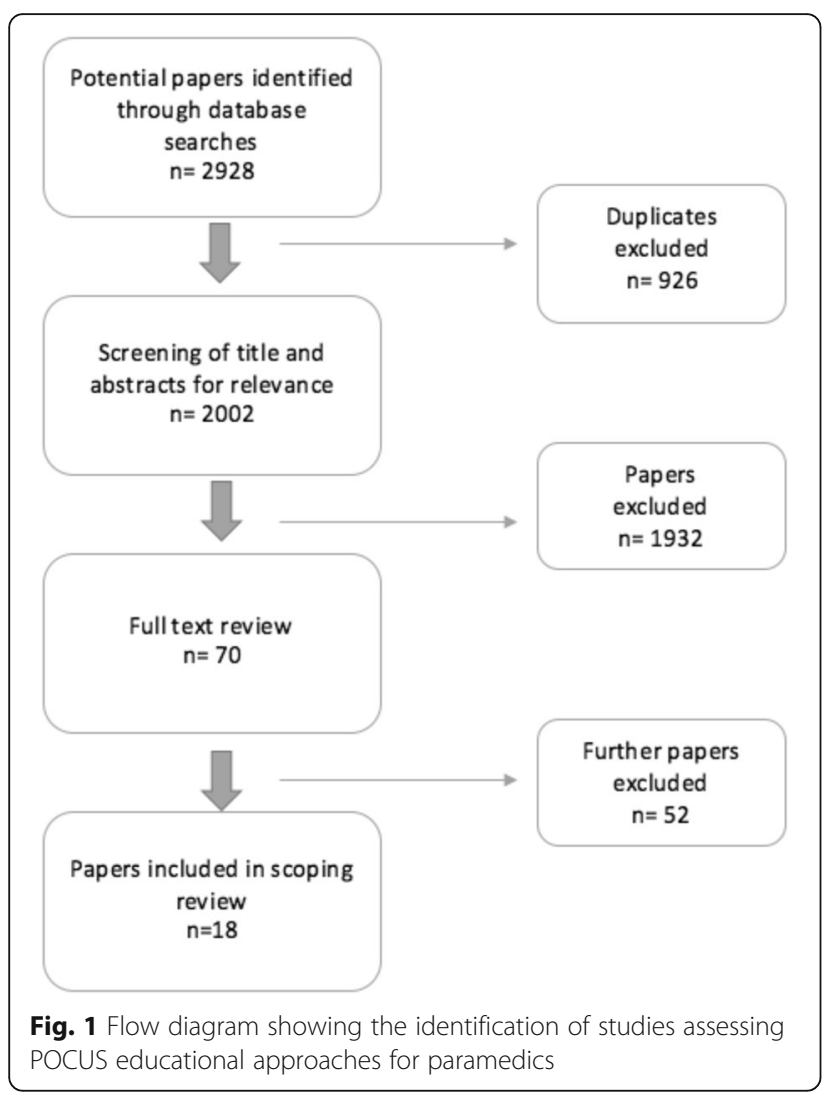

according to a common analytical framework [13]. The main areas of extracted data were participants, educational method (didactic, simulation, clinical), time and resource, and clinical condition researched.

5. Collating, summarising and reporting the results A total of 18 articles were included, comprising 11 prospective observational studies, 2 prospective educational intervention study, 1 single-blinded randomised controlled trial, 1 prospective educational cohort study, 1 prospective multi-center study, 1 blinded randomised controlled trial, and 1 combined retrospective and prospective observational study. Results are presented below and a summary is tabulated in Tables 2 and 3.

6. Consultation

Consultation was not included in this study due to time constraints.

\section{Results}

The initial search yielded 2002 de-duplicated articles. The titles of the relevant articles were screened for inclusion, and 1,984 were excluded as per the study protocol, see Fig. 1. The final inclusion consisted of 18 articles. Characteristics of the included studies are provided in Table 2 and the educational details of each study is provided in Table 3 .

\section{Participants professional backgrounds}

The population of participants undergoing POCUS training varied. Most articles reported combined cohorts of emergency providers with varying years of experience, however most were ultrasound naïve. Eight articles (44\%) described paramedic-only participants with small sample sizes of up to 20 [14-21]. One prospective cohort study described a larger paramedic population of 70 paramedics and 20 intermediate emergency medical technician (EMT) providers [22]. Five articles explored combined cohorts of flight paramedics and nurses [7, 8, 23-25]. One of these studies described 2 participants with prior, although limited, US exposure [7]. Only two articles reported on physicians and paramedics concurrently $[6,26]$. One study reported on a 'flight crew' but did not differentiate the professional backgrounds of participants [27].

\section{Clinical assessments}

There were six different clinical assessments for which paramedic POCUS curricula was explored, of which the FAST examination was the most common (Table 2).

\section{Clinical assessments and available curricula}

The curricula varied from two-minutes to two-days, with some studies also reporting ongoing training of up to six weeks (Table 3). The methods used ranged from online home based study to face-to-face lectures or instructional sessions. Didactic and practical sessions were reported in all studies, with only one study reporting an additional 1-h online home-based study program prior to initial training [22]. One study completed another educational session including a 90-min lecture and 90-min hands-on four months following initial training [25].

To determine the impact of the education multiple methods including written tests, recognition and interpretation of images, and objective structured clinical examinations (OSCEs) were used (Table 3). A pre-test was conducted in three studies to determine baseline knowledge with improved post-test scores (43\% vs $78 \%$, $78 \%$ vs $85 \%$, and $73 \%$ vs $95 \%$ respectively) following the educational program $[22,24,25]$.

The POCUS was for the majority performed in simulation and on healthy volunteers, see Table 2. Heegard et al. was the only article to include a further 8 -h of supervised US training on real patients in an emergency department [7]. Assessment for this study was conducted 6-weeks following initial training and involved a written test with an average score of $82 \%$ pre-training versus $71 \%$ when performed 12 -months later. Regarding the performance of the paramedics, of the seven articles that reported on accuracy measures, sensitivity ranged from 67 to $97.5 \%$, and specificity from 56 to 


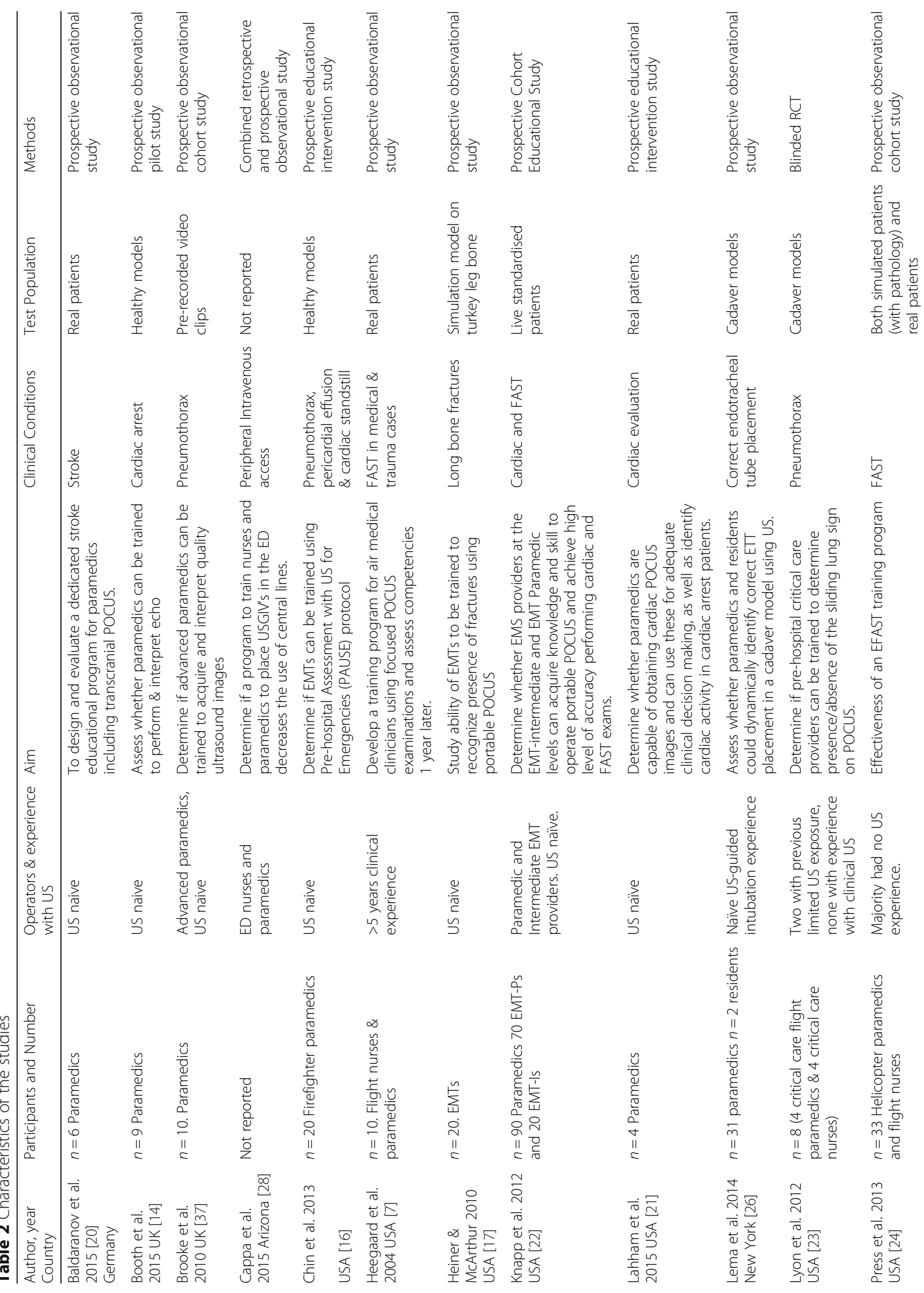




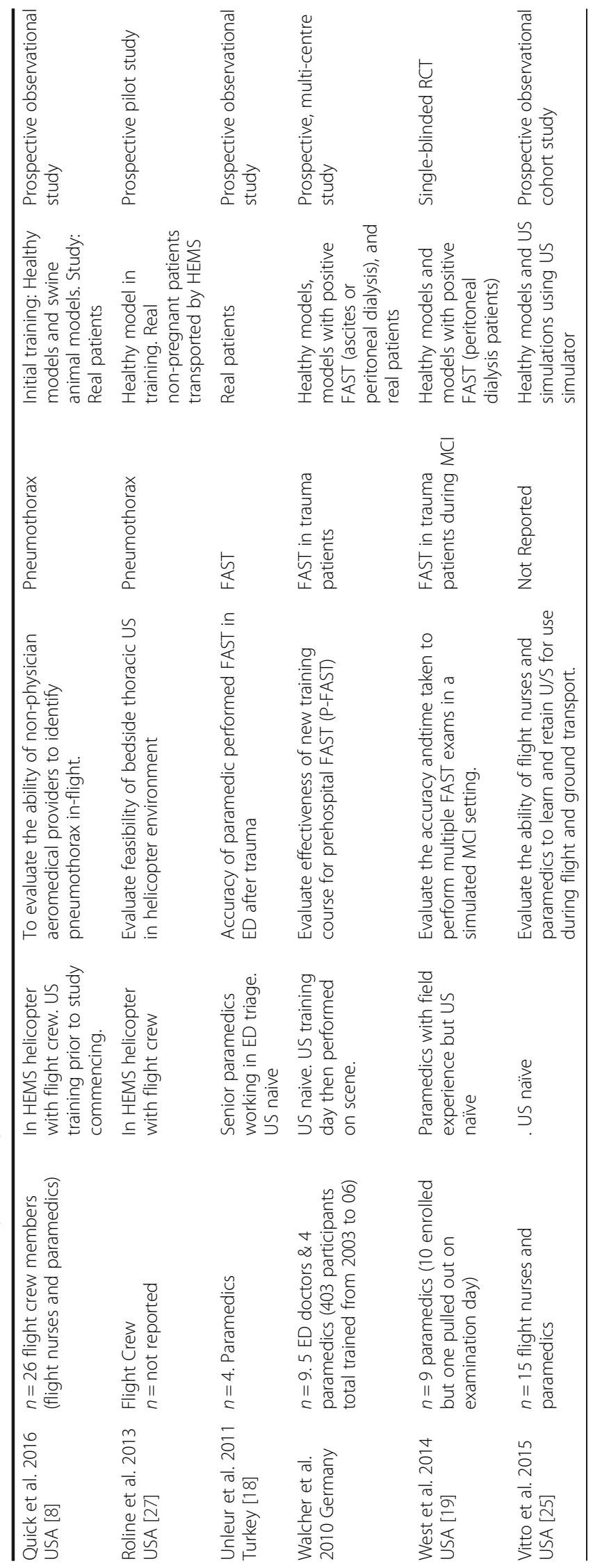




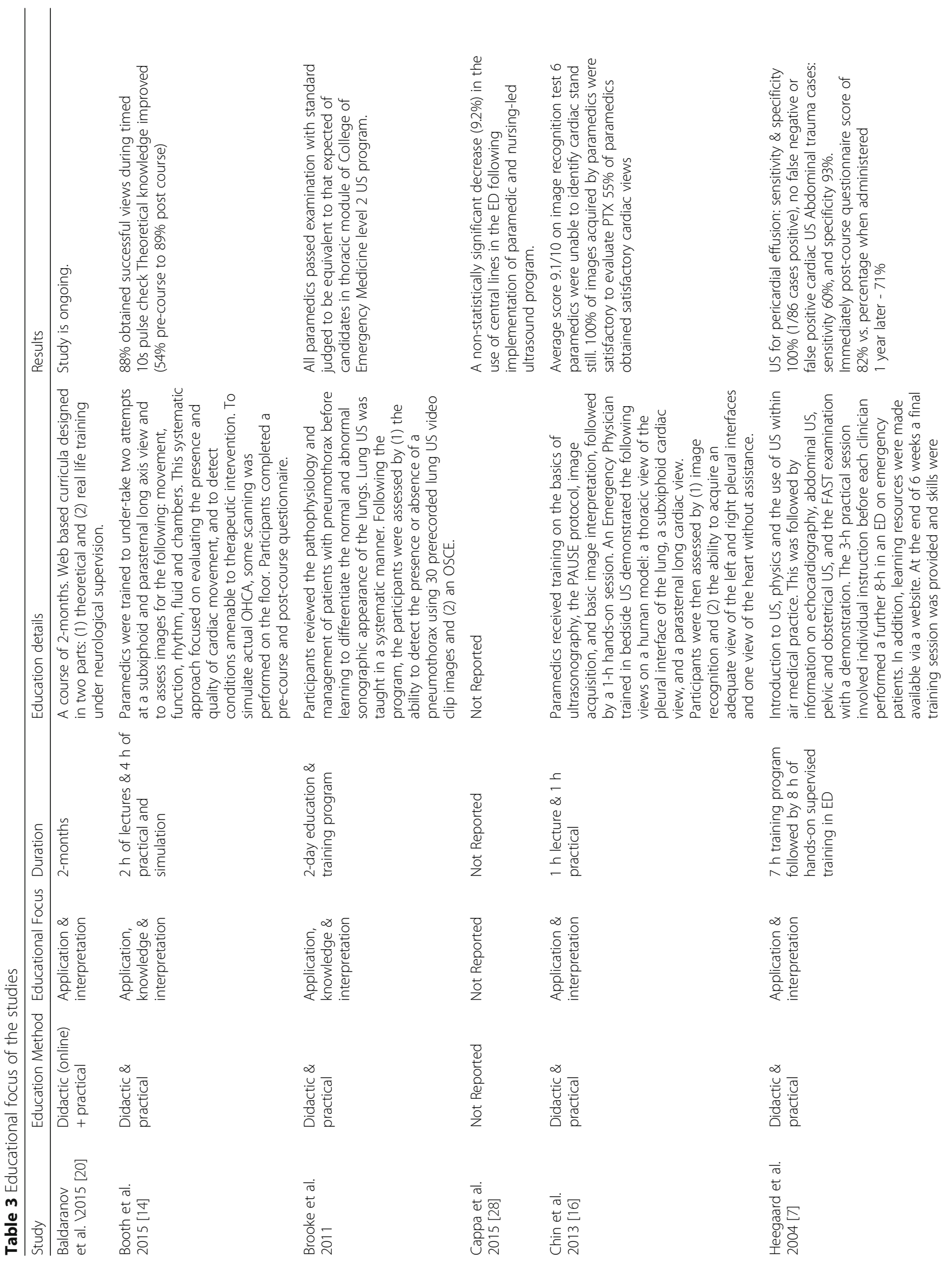




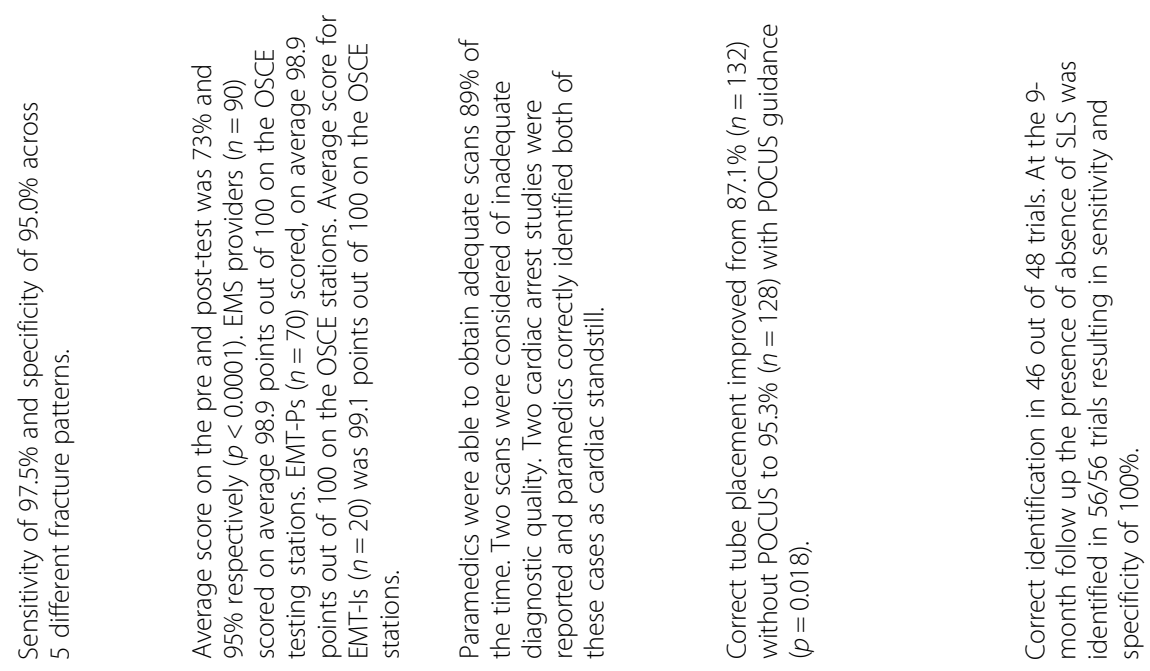

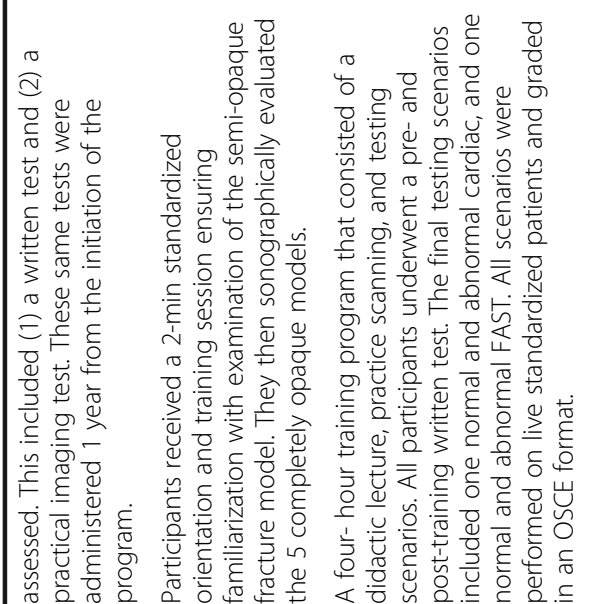

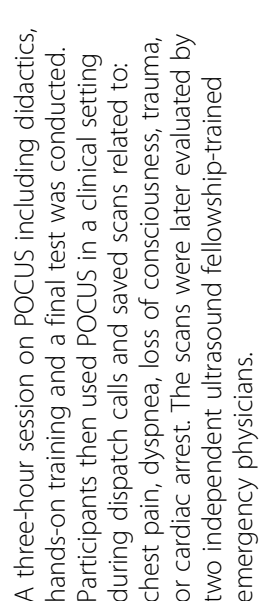

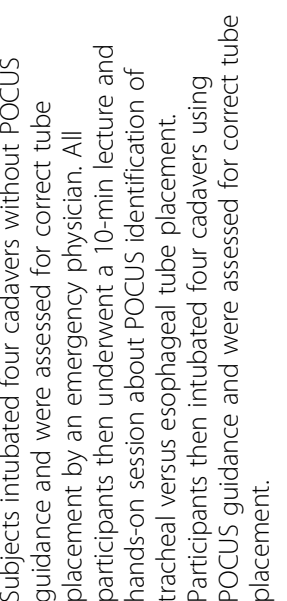

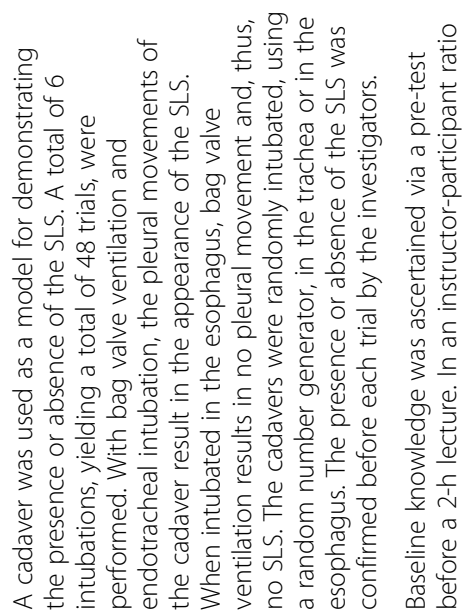
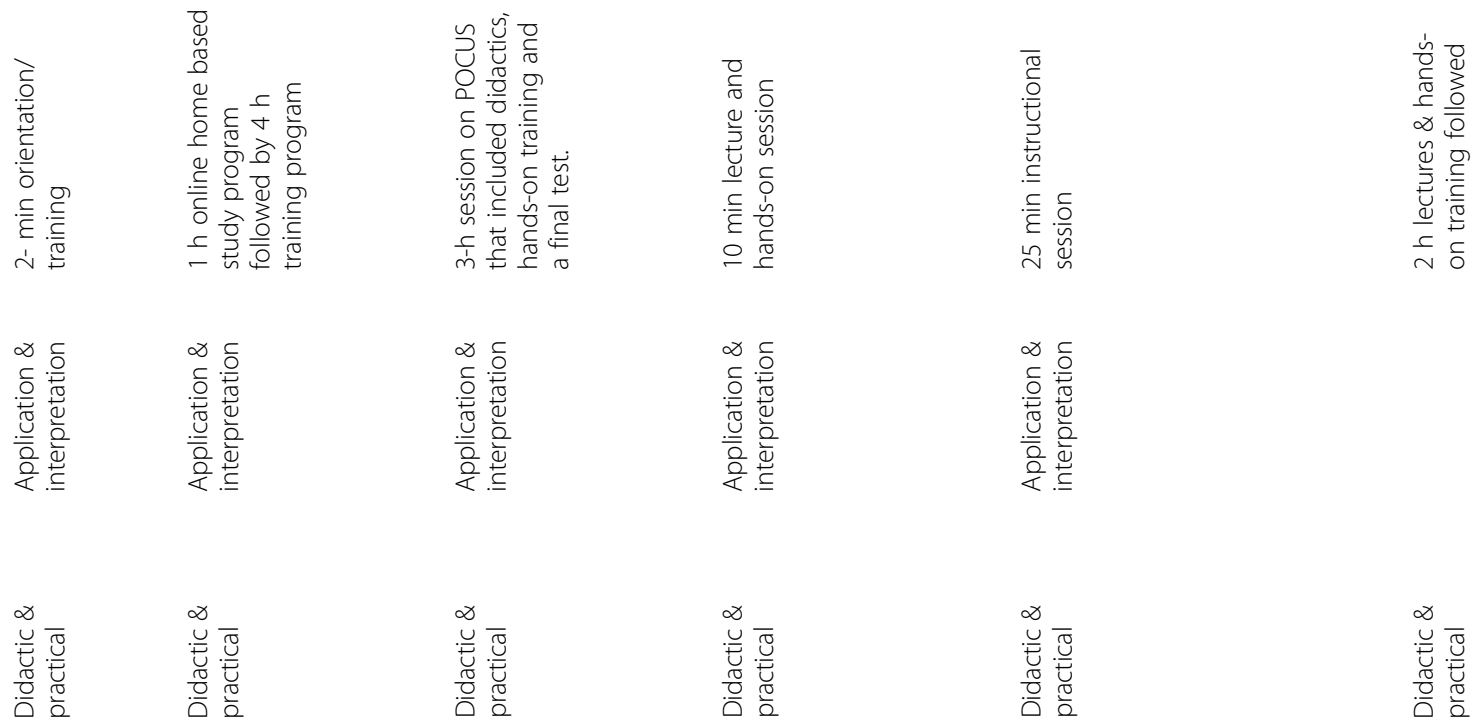

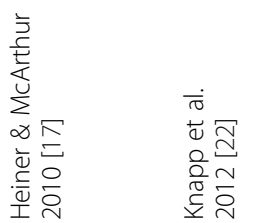
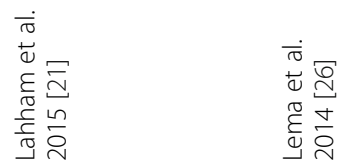

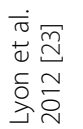

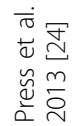




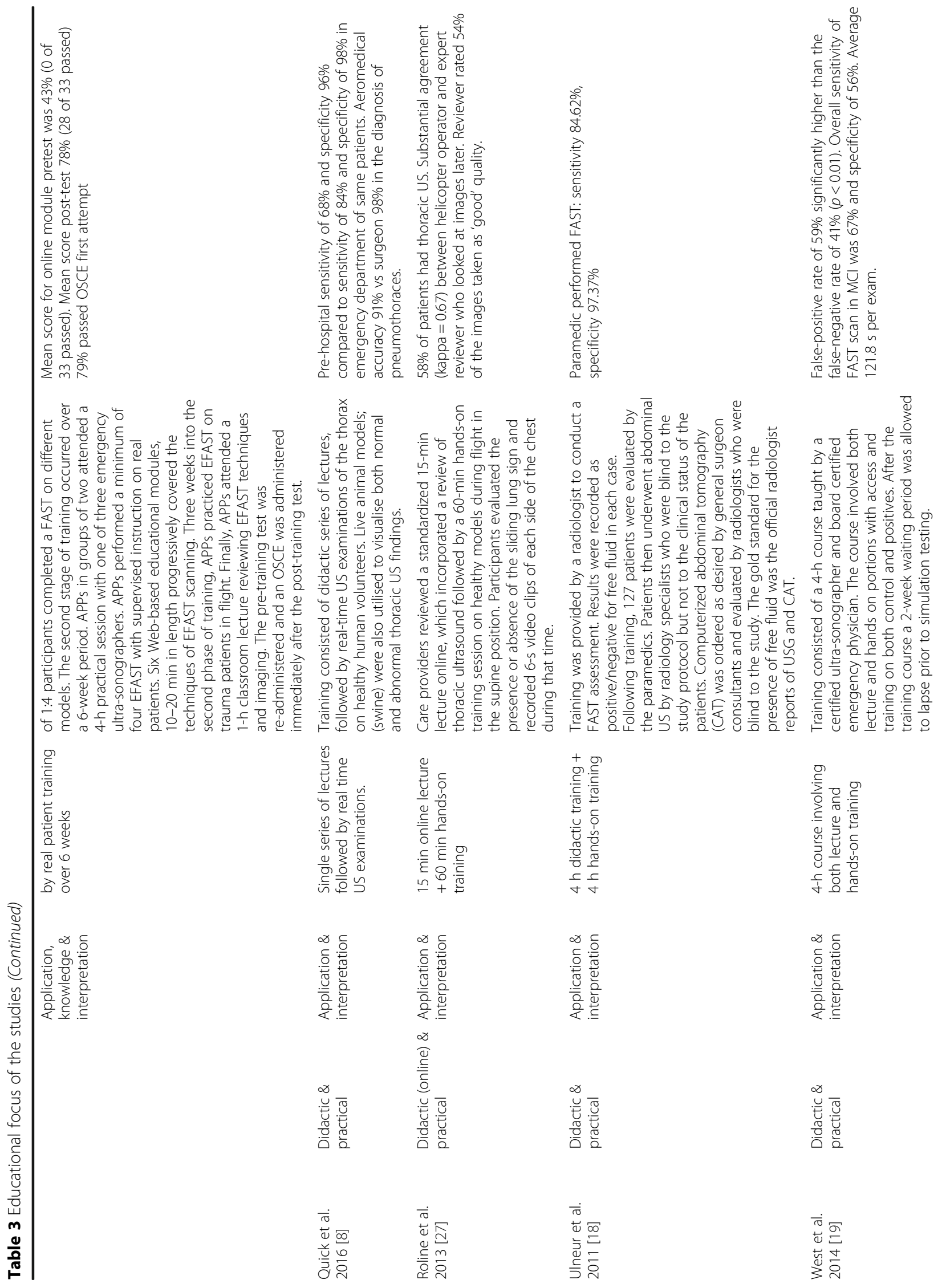




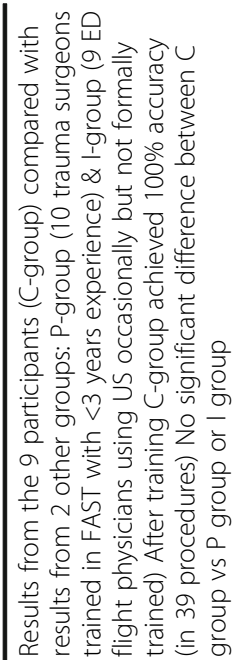

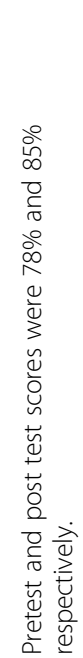

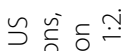

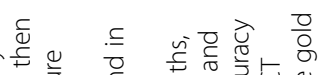

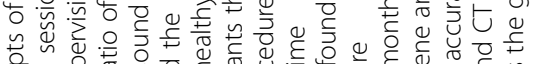

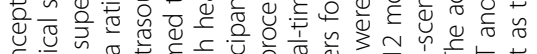

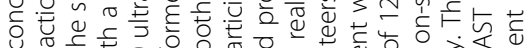

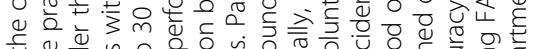

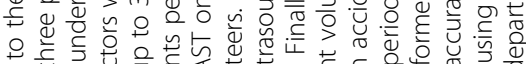

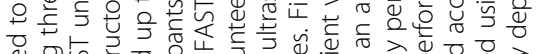

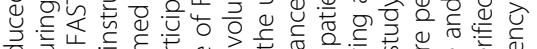

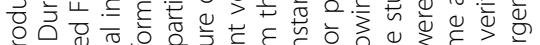

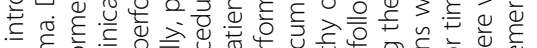
@

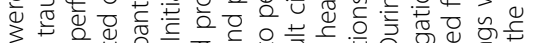
等.

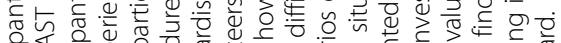

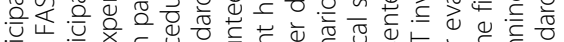

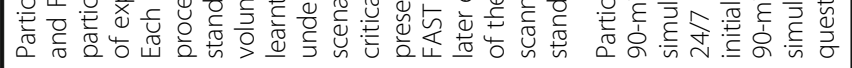

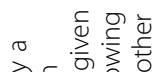

สิธ

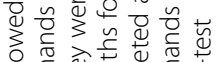

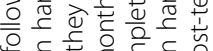

药艺

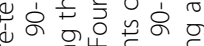

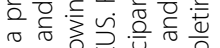

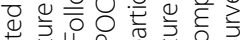

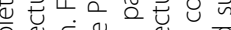

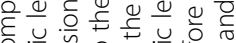
원

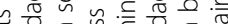

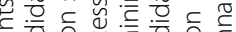
음

$\infty$ 
$97 \%[7,8,17-19,23,25]$. The lowest ranges related to a study in mass casualty incidents [19]. Results for a complete FAST examination was only reported by one author with a sensitivity of $84.6 \%$ and specificity of $97.3 \%$ [18]. One study specifically reported a sensitivity and specificity of $100 \%$ for pericardial effusion as well as a sensitivity of $60 \%$ and specificity of $93 \%$ for abdominal trauma cases [7].

The only study to report on the time taken to perform the assessment was the use of FAST in mass casualty incidents [19]. On average this took 121.8 s per examination [19].

Curricula for detecting pneumothoraces was described in four articles $[8,15,23,27]$. Duration varied with the shortest session a 25 -min combined instructional and practical program, and the longest a two-day program, see Table 3. All studies utilised didactic training followed by real-time practical ultrasound on either cadavers, healthy models, swine models, real patients or a combination of each. The sliding lung sign was described in three studies for identifying the presence or absence of pneumothorax [8, 24, 27], while one study also reported on comet tail artefacts, and seashore and stratosphere signs [15].

The sensitivity and specificity of POCUS for detecting pneumothoraces in the out-of-hospital setting following education was described by one study [8]. Quick et al. reported a sensitivity of $68 \%$ and a specificity of $84 \%$ compared to sensitivity of $84 \%$ and specificity of $98 \%$ in emergency department US of the same patients [8]. The accuracy of flight nurses and paramedics was found to be nearly as accurate (91\% vs $98 \%$ ) as the surgeon in the diagnosis of pneumothoraces [8]. At 9-months follow up Lyon et al. reported the highest sensitivity and specificity of $100 \%$ on a re-test for the identification of the sliding ling sign following a 25-min instructional video [23].

POCUS curricula for cardiac evaluation was explored in four articles [14, 16, 20, 21]. All studies included didactic and practical training, and duration varied from 2 -h to $6-\mathrm{h}$. Booth et al. reported $88 \%$ of paramedics obtained successful views on healthy patients with improved theoretical knowledge from $54 \%$ pre-course to $89 \%$ post-course [14]. Only one study reported in-field use of cardiac evaluation where paramedics were able to obtain adequate scans $89 \%$ of the time [21]. Two cardiac arrests were logged and paramedics correctly identified both cases as cardiac standstill. Chin et al. explored combined curricula of the heart and lungs to evaluate for pneumothorax, pericardial effusion and cardiac activity [16]. An average test score of 9.1/10 on image recognition was reported, however 6 paramedics were unable to identify cardiac standstill.

POCUS curricula for long bone fracture recognition was reported by one study [17]. A fracture simulation model was composed of five different types of mechanically fractured bare turkey leg bone. Paramedic participants received a 2-min standard orientation and training session prior to evaluation. Across all fracture patterns the paramedics achieved an overall detection sensitivity of $97.5 \%$ and specificity of $95.0 \%$ [17].

Only one study reported on the POCUS curricula in the use of stroke assessment over a two-month period including theoretical training and real life training under neurological supervision [20]. This study remains ongoing and results are yet to be reported. Similarly, only one article explored POCUS curricula for peripheral intravenous access to determine if this decreases the use of central lines in the ED [28].

The use of POCUS to identify correct endotracheal tube placement was reported by one article [26]. In this study all participants intubated cadaver models without POCUS guidance followed by a 10-min lecture and hands-on training. Participants then intubated four cadaver models using POCUS guidance. Correct tube placement improved from $87.1 \%$ without POCUS guidance to $95.3 \%(P=0.018)$ with POCUS guidance.

\section{Study location}

Most of the studies were conducted in the United States (13 studies), while the remaining studies were undertaken in other countries including the United Kingdom (2 studies), Germany (2 studies) and Turkey (1 study), see Table 2.

\section{Discussion}

Ultrasound is a relatively new and emerging assessment modality in out-of-hospital care, especially for paramedics. Technology has progressed to the point where small hand-held devices are readily available for out-ofhospital use. This scoping review examined 18 articles to describe the different curricula used for training paramedics in POCUS in the out-of-hospital setting.

The professional backgrounds of participants undergoing out-of-hospital POCUS training varies. Most studies reported on combined cohorts of physicians, nurses and paramedics all with varying years of experience. Knowledge of gross and functional anatomy can vary significantly, dependent on individual professional background (if any), and the duration and quality of initial education. Numerous physician-based prehospital services across the developed world have been using prehospital US for many years and are mostly helicopter emergency medical services (HEMS) [29, 30]. Physicians staffing EMS prehospital systems are usually sourced from the specialties of anaesthesia, intensive care and emergency medicine. Thus, these staff will already have undertaken significant education in gross and functional anatomy during undergraduate medical studies, and will likely 
have at least basic training in POCUS as part of their specialist qualification.

Conversely, paramedic regulation and registration as health professionals varies across the world, and there is no standardised qualification to be termed a paramedic in many jurisdictions [31]. The base qualifications for paramedics working in out-of-hospital systems in developed nations may range from short vocational courses, or undergraduate degrees through to postgraduate-level courses. Therefore, it is intuitive that experienced practitioners would gain and achieve competency in a skill dependent on anatomical knowledge more readily than less-experienced colleagues. In this study, paramedics with advanced training and/or extensive experience demonstrated a higher degree of accuracy in POCUS interpretation (where measured) [23]. However, the data available at this time does not show a trend towards a difference between the groups, and a correlation to clinical outcomes is not able to be demonstrated, and is beyond the scope of this study.

This scoping review suggests that the curricula used has varied considerably. Training duration ranged from a short 2-min orientation session to two days with most sessions completed over a one-day course. Of interest, the literature reports POCUS curricula being implemented for physicians as early as the first year of medical school [32]. In one study, Golgalniceanu et al. enrolled third and fifth year students in a 5-h FAST course of which $85 \%$ of students completed a full FAST scan at an adequate level of performance in under 6-min [33]. Should further studies demonstrate clear clinical utility of prehospital POCUS, then it may be reasonable to commence basic training in ultrasound during undergraduate paramedic training. Introduction of the skill early in the career of paramedics may strengthen anatomical knowledge, and allow for development of competence in POCUS over an extended period.

Blended learning that integrates face-to-face learning with computer-mediated online components has recently been reported as an effective approach for efficient learning and skill acquisition in POCUS [32, 34]. In this scoping review only two studies included an additional online component $[22,27]$. Knapp et al. reported improved posttest scores from 73 to $95 \%$ with EMS providers scoring 98.9 to $99.1 \%$ on the OSCE stations [22]. This suggests online learning may assist with proficiency in learning the skills and knowledge required for performing POCUS, however is yet to be demonstrated as a superior learning tool. Simulation training for skill acquisition is also well documented in the literature [32, 35]. Interestingly, nine of the studies in this review were performed solely in the classroom on healthy volunteers or cadavers $[6,14-17,19,23,25,26]$. The remaining studies were performed on ill or injured patients in clinical practice. Further research is required to demonstrate which method provides greater outcomes in regards to clinical education.

At the commencement of training for the various groups, strict protocol for the clinical application of POCUS was not always defined. After training was completed, actual POCUS utilisation varied from FAST, pneumothoraces, cardiac evaluation or a combination of these. The study by Chin et al. was robust in that it followed a strict protocol, although that protocol was limited to only cardiac and lung ultrasound [16]. Nevertheless, adhering to a structured, measured and comprehensive training program, and then instituting a systematic protocol for in-field application of POCUS allowed for ready analysis of outcomes. These inconsistencies may translate to difficulty in assessing clinical utility of out-of-hospital POCUS, but also how proficient paramedics were in interpreting images, and advancing their psychomotor skills.

Paramedic interpretation of ultrasound images in most studies was "confirmed" by US experts. However, in one study, there was no "closing of the loop" with regard to the learning process [18]. Correlation with expert interpretation would seem prudent, given the novel and relatively complex nature of POCUS in the out-of-hospital environment. This highlights the importance of studies examining paramedic education and proficiency in out-ofhospital POCUS which should include assessment of paramedic-acquired images by ultrasound experts, such as sonographers and/or experienced critical care physicians.

Despite various education approaches our review suggests that paramedics may be able to gain proficiency in POCUS reasonably promptly, regardless of base qualification, experience, duration or perceived quality of training. In the study by Quick et al., a small group of paramedics with (on average) greater than ten years' experience, who underwent a comprehensive education and training program adhered to a strong study protocol and had a high level of patients exposure were able to demonstrate POCUS interpretation accuracy similar to that of trauma surgeons [8]. These data correlate with other studies where paramedics have initiated critical care procedures historically only performed by physicians (in and out-of-hospital) [36]. This suggests that a short duration of education and subsequent continuing clinical exposure (of which a minimum standard is difficult to determine) may be sufficient to enable paramedics to remain proficient in out-of-hospital POCUS. This concept requires further rigorous investigation through well-defined prospective clinical trials.

\section{Future directions}

At this time, clinical utility of POCUS, especially in the hands of paramedic clinicians, has been difficult to 
demonstrate [37]. Further investigations may consider comparing basic out-of-hospital providers with critical care practitioners, who undergo a standardised POCUS education process. Such a study should examine not only experience of the clinician and the ability to interpret images, but also the level of exposure to the skill, and subsequent clinical application of information gained from POCUS exam.

A multi-center study, utilising a standardised education process with confirmation of image interpretation would be valuable to accurately measure paramedic proficiency. Additionally, such a study should assess variables including time taken to perform the scan, diagnosis and patient outcomes, with a view to evaluate clinical utility, and quantify the learning process. Other studies comparing solely classroom-based training versus live patient training, or a combination, would be useful in determining the most effective way to educate paramedics in the use of POCUS. In this scoping review, the studies did not compare a standard POCUS assessment. Future studies, such as a suggested standardised multi-center trial, should include standardised assessment so that accurate comparisons between groups can be made.

\section{Limitations}

The authors acknowledge the limitations of the scoping review methodology. BM, NR and TSc are all operational paramedics utilising POCUS in their critical care practice, thus there is an acknowledged risk of bias in article selection and interpretation. This was mitigated by including authors that have limited or no experience in the application of out-of-hospital POCUS.

\section{Conclusions}

POCUS curricula for paramedics in the out-of-hospital setting varies considerably. There appears to be no consistent approach, with some systems not quantifying or revising quality of learning. Paramedics with different levels of training and experience appear to be able to gain skills in POCUS, however correlation with clinical utility is difficult to measure. Furthermore, a lack of standardised training and a structured clinical application process makes it difficult to evaluate POCUS as a useful modality in the provision of out-of-hospital care. Further studies investigating education for paramedic POCUS and subsequent clinical application should include a standardised education process, expert assessment of paramedic competency, and formalised protocols for the application of out-ofhospital POCUS, so as to measure clinical utility.

\section{Abbreviations}

eFAST: extended Focused Assessment Sonography for Trauma; EMS: Emergency Medical Service(s); EMT: Emergency Medical Technician; FAST: Focused Assessment Sonography for Trauma; HEMS: Helicopter
Emergency Medical Services; OSCEs: Objective Structured Clinical Examinations; POCUS: Point-of-care ultrasound

Acknowledgements

Not applicable.

Funding

Funding was not sought for this research project.

Availability of data and materials

All data generated or analysed during this study are included in this published article.

\section{Authors' contributions}

$\mathrm{BM}, \mathrm{AO}$ and $\mathrm{BW}$ conceived the study design. $\mathrm{AO}$ performed the initial database search. Duplicates were removed by BW. Titles and abstracts were appraised for eligibility by five independent authors (BM, NR, TSC, CM and $\mathrm{BW}$. $\mathrm{AD}, \mathrm{BM}, \mathrm{AO}$ and $\mathrm{BW}$ completed the review and analysis. All authors contributed to the final manuscript. All authors read and approved the final manuscript.

\section{Competing interests}

The authors declare that they have no competing interests.

Consent for publication

Not applicable.

Ethics approval and consent to participate

Not applicable.

\section{Publisher's Note}

Springer Nature remains neutral with regard to jurisdictional claims in published maps and institutional affiliations.

Received: 16 March 2017 Accepted: 12 June 2017

Published online: 17 June 2017

\section{References}

1. Davison K, Forbes MP. Pre-hospital medicine: A glimpse of the future. Australasian J Paramedicine. 2015;12:5.

2. El Sayed MJ, Zaghrini E. Prehospital Emergency Ultrasound: A Review of Current Clinical Applications, Challenges, and Future Implications. Emergency Medicine International. 2013. http://dx.doi.org/10.1155/2013/531674

3. Jaffer U, Aslam M, Jaffer A, Standfield N, Pennell A. Emergency ultrasound in the prehospital setting. J Paramed Pract. 2011;3:83-6.

4. Nelson BP, Chason K. Use of ultrasound by emergency medical services: a review. Int J Emerg Med. 2008;1:253-9.

5. Nakahara S, Matsuoka T, Ueno M, Mizushima Y, Ichikawa M, Yokota J, Yoshida K. Predictive factors for undertriage among severe blunt trauma patients: what enables them to slip through an established trauma triage protocol? J Trauma Acute Care Surg. 2010;68:1044-51.

6. Walcher F, Weinlich M, Conrad G, Schweigkofler U, Breitkreutz R, Kirschning T, Marzi I. Prehospital ultrasound imaging improves management of abdominal trauma. Br J Surg. 2006;93:238-42.

7. Heegaard W, Plummer D, Dries D, Frascone R, Pippert G, Steel D, Clinton J. Ultrasound for the air medical clinician. Air Med J. 2004;23:20-23.

8. Quick JA, Uhlich RM, Ahmad S, Barnes SL, Coughenour JP. In-flight ultrasound identification of pneumothorax. Emerg Radiol. 2016;23:3-7.

9. McCallum J, Vu E, Sweet D, Kanji HD. Assessment of paramedic ultrasound curricula: A systematic review. Air Med J. 2015;34:360-8.

10. Aylwin CJ, Brohi K, Davies GD, Walsh MS. Pre-hospital and In-hospital thoracostomy: indications and complications. Ann R Coll Surg Engl. 2008;90:54-7.

11. Davis K, Drey N, Gould D. What are scoping studies? A review of the nursing literature. Int J Nurs Stud. 2009;46:1386-400.

12. Levac D, Colquhoun H, O'Brien KK. Scoping studies: advancing the methodology. Implement Sci. 2010;5:1.

13. Arksey H, O'Malley L. Scoping studies: towards a methodological framework. Int J Soc Res Methodol. 2005;8:19-32.

14. Booth KL, Reed MJ, Brady S, Cranfield KR, Kishen R, Letham K, McAlpine G, Murray DB, Wilson EB, Wilson M, Short S. Training paramedics in focussed echo in life support. Eur J Emerg Med. 2015;22:430-5. 
15. Brooke M, Walton J, Scutt D, Connolly J, Jarman B. Acquisition and interpretation of focused diagnostic ultrasound images by ultrasound-naive advanced paramedics: trialling a PHUS education programme. Emerg Med J. 2012:29:322-6.

16. Chin EJ, Chan CH, Mortazavi R, Anderson CL, Kahn CA, Summers S, Fox JC. A pilot study examining the viability of a prehospital assessment with ultrasound for emergencies (PAUSE) protocol. J Emerg Med. 2013;44:142-9.

17. Heiner JD, MCArthur TJ. The ultrasound identification of simulated long bone fractures by prehospital providers. Wilderness Environ Med. 2010;21:137-40.

18. Unlüer EE, Yavaşi O, Kara PH, Klılı̧ TY, Vandenberk N, Kayayurt K, Kıyançiçek S, Akoğlu H, Yılmaz C. Paramedic-performed focused assessment with sonography in trauma (FAST) in the emergency department. Turkish J Trauma Emerg Surg. 2011;17:113-6.

19. West B, Cusser A, Etengoff S, Landsgaard H, LaBond V. The use of fast scan by paramedics in mass-casualty incidents: A simulation study. Prehospital and Disaster Med. 2014;29:576.

20. Baldaranov D, Kilic M, Pflug K, Theiss S, Leis A, Pemmerl J, Pels H, Boy S, Bogdahn U, Schlachetzki F. Prehospital stroke education in paramedics. Eur J Neurol. 2015;22:799.

21. Lahham S, Rooney K, Sloane B, Fox JC. Prehospital assessment with ultrasound In emergencies-pause II. Acad Emerg Med. 2015;22:S227.

22. Knapp B, Byars D, Stewart V, Ryszkiewicz R, Evans D. Emergency medical services focused assessment with sonography in trauma and cardiac ultrasound in cardiac arrest: the training phase. Acad Emerg Med. 2012;19:S189.

23. Lyon $M$, Walton $P$, Bhalla $V$, Shiver SA. Ultrasound detection of the sliding lung sign by prehospital critical care providers. Am J Emerg Med. 2012;30:485-8.

24. Press GM, Miller SK, Hassan IA, Blankenship R, del Junco D, Camp E, Holcomb JB. Evaluation of a training curriculum for prehospital trauma ultrasound. J Emerg Med. 2013;45:856-64.

25. Vitto MJ, Spector D, Evans DP. Implementation of a flight medical crew ultrasound training program. Acad Emerg Med. 2015;22:S133.

26. Lema PC, Wilson J, O'brien M, Lindstrom H, Tanski C, Consiglio J, Clemency B. Ultrasound identification of successful endotracheal tube placement by paramedics and residents. Ann Emerg Med. 2014;64:S8.

27. Roline CE, Heegaard WG, Moore JC, Joing SA, Hildebrandt DA, Biros MH, Caroon LV, Plummer DW, Reardon RF. Feasibility of bedside thoracic ultrasound in the helicopter emergency medical services setting. Air Med J. 2013;32:153-7

28. Cappa AR, Minckler MR, Wyatt RG, Binger CW, Stolz U, Amini R, Adhikari S, Stolz L. 291 effect of ultrasound-guided peripheral intravenous catheter placement by nurses and paramedics on central line placement in the emergency department. Ann Emerg Med. 2015;66:S105.

29. Noble VE, Lamhaut L, Capp R, Bosson N, Liteplo A, Marx JS, Carli P. Evaluation of a thoracic ultrasound training module for the detection of pneumothorax and pulmonary edema by prehospital physician care providers. BMC Med Educ. 2009:9:3.

30. Lapostolle F, Petrovic T, Lenoir G, Catineau J, Galinski M, Metzger J, Chanzy E, Adnet F. Usefulness of hand-held ultrasound devices in out-of-hospital diagnosis performed by emergency physicians. Am J Emerg Med. 2006;24:237-42.

31. Knox S. International and unifying standards of pre-hospital care. Irish J Paramed. 2016;1:2

32. Lewiss RE, Hoffmann B, Beaulieu Y, Phelan MB. Point-of-care ultrasound education. J Ultrasound Med. 2014;33:27-32.

33. Gogalniceanu P, Sheena Y, Kashef E, Purkayastha S, Darzi A, Paraskeva P. Is basic emergency ultrasound training feasible as part of standard undergraduate medical education? J Surg Educ. 2010;67:152-6.

34. Mendiratta-Lala M, Williams T, De Quadros N, Bonnett J, Mendiratta V. The use of a simulation center to improve resident proficiency in performing ultrasound-guided procedures. Acad Radiol. 2010;17:535-40.

35. Wang EE, Quinones J, Fitch MT. Developing technical expertise in emergency medicine: The role of simulation in Procedural skill acquisition. Acad Emerg Med. 2008;15:1046-57.

36. Bernard SA, Nguyen V, Cameron P, Masci K, Fitzgerald M, Cooper D, Walker T, Myles P, Murray L, David T, Smith K, Patric I, Edington J, Bacon A, Rosenfield J, Judson R. Prehospital rapid sequence intubation improves functional outcome for patients with severe traumatic brain injury: A randomised controlled trial. Ann Surg. 2010;252:959-65.

37. Brooke M, Walton J, Scutt D, Connolly J. Paramedic application of ultrasound in the management of patients in the prehospital setting: a review of the literature. Emerg Med J. 2010;27:702-7.

\section{Submit your next manuscript to BioMed Central and we will help you at every step:}

- We accept pre-submission inquiries

- Our selector tool helps you to find the most relevant journal

- We provide round the clock customer support

- Convenient online submission

- Thorough peer review

- Inclusion in PubMed and all major indexing services

- Maximum visibility for your research

Submit your manuscript at www.biomedcentral.com/submit
Biomed Central 